\title{
Evaluation of Light and Color Performances of Deep Black Coloring of Non Circular Cross Section Polyester Fabrics using Polarization Image Processing
}

\author{
Xiaosong Liu ${ }^{1,3 *}$, Fumei Wang ${ }^{1,2}$ and Ian $\mathbf{R}$ Hardin $^{3}$ \\ ${ }^{1}$ Department of Textiles, Merchandising, and Interiors, University of Georgia, Athens, USA \\ ${ }^{2}$ Key Lab of Textile Science and Technology Ministry of Education, Shanghai, China
}

${ }^{3}$ College of Textiles, Donghua University, Shanghai, China

\begin{abstract}
The light and color performances of deep-black-coloring of fabrics made of Non-Circular Cross-Section (NCCS) Polyethylene Terephthalate (PET) fibers were investigated by means of GP, SEM and polarization imaging. The GP surface reflectance curves showed that the performance of single NCCS fibers were profoundly affected by the forces loaded under horizontal and vertical directions compared to its yarns. The unique surface characteristics of the NCCS PET fabrics indicated by its constituent fibers' cross-sections and fabrics' SEM images gave unpredictable effects on the light and color perceived on the deep-coloring effect of the fabrics. Polarization imaging results of black-dyed woven fabrics indicated that higher specular and chroma values affected the deep-coloring effect, and that the overlapping chroma curves means that there were insignificant differences among those black fabrics. Meanwhile the similar results for the knitted fabrics suggested that higher values of chroma means higher effect of deep-coloring compared to undyed/white samples with higher values of specular reflectance and shine. Subjective visual evaluation was carried out on the post-processed polarization images of the NCCS PET woven samples and was compared to the instrumental results. The results revealed that there was good consistency on deep-coloring perceived between the intensity images and average value of chroma while inconsistency occurred between the intensity images and the average value of intensity.
\end{abstract}

Keywords: Non-circular cross-section (NCCS); Polyester fiber; Surface reflectance, Goniophotometer (GP); Polarization image processing

\section{Introduction}

The perceived color of an object is affected by three components: the physical and chemical composition of the object, the spectral composition of the light source illuminating the object, and the spectral sensitivity of the viewers' eyes. The color of an object can vary in three dimensions, namely the hue-typically the "color" of the object, lightness, the brightness of the object and saturation-which is the purity or chroma of the color. These can be put into a mathematical color system such as RGB or CIE [1]. The perceived colors can be affected by variations caused by the gender [2-4] and aging [5] of participants. The instrumental and human subjective evaluation of color will be affected by the gloss, and translucency of the object, taking the reflected and diffused light into account. The instrument sees only light reflected from a limited solid angle, while the human eye is influenced by multidirectional illumination, which contributes to the difference between the two types of evaluations.

A mathematical and geometric model of deep-dyeing or deepcolouring was developed based on the "pile of plates" idea for analysing the colour of fabrics. It was assumed that each "plate" consists of a parallel array of isotropic cylinders of equal diameter with uniform dye distribution, surrounded by a transparent and continuous medium $[5,6]$. This proved to be effective for dyed circular fibers by taking the ring dyeing phenomenon [7,8] and internal scattering [9] into consideration. However, this model only works in cylindrical or round fibers and ideal fabrics. It neglects the reflection-refraction pattern of neighbouring fibers [7-10]. Other factors, such as synthetic fiber translucency [11], dye distribution [6,7,10], unique numerical simulation method [12] and the moisture condition of the textiles by an alternative approach based on that of Goldfinger [9]. These should also be taken into account when discussing the color yield. The only common factor in all of the aforementioned research was the calculation method applied to cylindrical fibers with one cross section feature like a circular arc.

Other works $[13,14]$ showed that the depth of shade depends mainly on the fiber denier, the inherent colours of the fiber, its surface and its geometry. These two papers were mostly based on experimental testing results and concluded that the depth of shade would be changed as the cross-section shapes varied. There was doubt that the mathematical relationship and index FI be universal and effective for other fibers with unique cross-section shapes. However, the variations of different NCCS fibers are innumerable, which means that the previous model and its alternative one should be reconstructed to match the real or quasiactual conditions of NCCS fibers, their yarns, and even fabrics.

Polarization is a well known technique to carefully analyze the composition of the light scattered by an object [15-18]. If the illumination is polarized, the reflected and refracted light will remain polarized while the scattered light will be depolarized, and this fundamental property allows measuring independently the diffused and the reflected light.

*Corresponding author: Xiaosong Liu, Department of Textiles, Merchandising and Interiors, University of Georgia, Athens, GA 30602, USA, Tel. 706-583-0172 E-mail: xiaosongliu7405@aliyun.com

Received November 15, 2013; Accepted December 06, 2013; Published December 13, 2013

Citation: Liu X, Wang F, Hardin IR (2013) Evaluation of Light and Color Performances of Deep Black Coloring of Non Circular Cross Section Polyester Fabrics using Polarization Image Processing. J Textile Sci Eng 4: 145. doi:10.4172/2165-8064.1000145

Copyright: ( 2013 Liu X, et al. This is an open-access article distributed under the terms of the Creative Commons Attribution License, which permits unrestricted use, distribution, and reproduction in any medium, provided the original author and source are credited. 
Polarization imaging can be used for characterizing hair luster [20] and color vibrance [21], fiber orientation [22] and birefringence [20,23].

In this work, six woven and knitted fabrics made of Non-Circular Cross Section (NCCS) Polyester (PET) filaments were dyed, and polarization imaging was applied to characterize the color information. The surface reflection of NCCS PET yarns and their constituent monofilaments were investigated by using a Goniophotometer (GP) for analyzing the relationship between the light reflectance and the color of the samples being tested. Subjective evaluation was carried on the polarization imaging posted images to examine the consistency with that of the instrumental results. All these were done in order to find out whether the NCCS PET fabrics would show deep-coloring and how this is related to the NCCS fiber used.

\section{Materials and Methods}

\section{Fiber and fabrics}

Two comparable sets in linear density around 120 and 210 dtex of NCCS PET full drawing yarns (FDY) made of the semi-dull PET slices were conducted for comparing the light properties. The specifications of the different NCCS filaments are shown in Table 1.

BBecause there are many grooves on their surfaces, the NCCS filament yarns cannot be used as warp yarns through sizing, because the sizing agents have unpredictable effects on the optical performance. To avoid this impact, the NCCS PET filament yarns were used as weft yarns to produce a suitable fabric structure with as much of the NCCS PET filament yarns on the surface of the fabric as possible. Circular cross section PET filaments with $120 \mathrm{D} / 96 \mathrm{~F}$ and twist of $1100 \mathrm{~T} / \mathrm{m}$ (S direction) were used in the warp yarn. The fabric parameters were calculated based on the covering factor [24] in warp and weft directions as ET, EW and the total one $\mathrm{E}$ with the value of $52.1 \%, 77.6 \%$ and $89.3 \%$, respectively. The weaving structure was $5 / 3$ weft stain and the yarn density $(\mathrm{ends} / 10 \mathrm{~cm}$ ) in warp and weft directions are 405 ends and 606 picks, respectively. The knitted fabric samples were made of Y1 and Y3 yarns, respectively, by using 3.5-inch lab-scaled knitting machine.

Two knitted samples marked as K1 and K2 was made of Y1 yarn for testing the effect of the undyed and dyed color on the polarization image processing results. The third knitted sample $\mathrm{K} 3$ was made of $\mathrm{Y} 3$ yarn for figuring out the effect of filament's fineness on its polarization image processing results, compared to that of K1.

\section{Pre-treatment and dying procedure of fabric samples}

To reduce the impacts of natural impurities and spinning oil on the light reflectance of polyester filaments, a pure hot water pretreatment was applied to the fibers and fabrics in a KQ-500VDE Type ultrasonic cleaner with the following parameters:temp $=60^{\circ} \mathrm{C}, \mathrm{t}=30 \mathrm{~min}$, freqency $=45 \mathrm{khz}$, power $=100 \mathrm{w}$. Before being tested, the fabrics were placed inside a room with ambient temperature at $25 \pm 2^{\circ} \mathrm{C}$ and $60 \pm 3 \%$ relative humidity for 24 hours.

\begin{tabular}{|c|c|c|c|c|}
\hline Yarn No. & Fineness/dtex & $\begin{array}{c}\text { Filament } \\
\text { Number }\end{array}$ & $\begin{array}{c}\text { cross section } \\
\text { type }\end{array}$ & Fabrics No. \\
\hline Y1 $^{\#}$ & 126.21 & & & $\mathrm{~F}^{\#}$ \\
\hline Y2 $^{\#}$ & 120.98 & & & $\mathrm{~F}^{\#}$ \\
\hline Y3 $^{\#}$ & 215.57 & 36 & NCCS & $\mathrm{F}^{\#}$ \\
\hline Y4 $^{\#}$ & 207.37 & FDY & & $\mathrm{F}^{\#}$ \\
\hline Y5 $^{\#}$ & 118.13 & & round & $\mathrm{F5}^{\#}$ \\
\hline Y6 $^{\#}$ & 208.67 & & & $\mathrm{F6}^{\#}$ \\
\hline
\end{tabular}

Table 1: Specifications of different NCCS filaments.

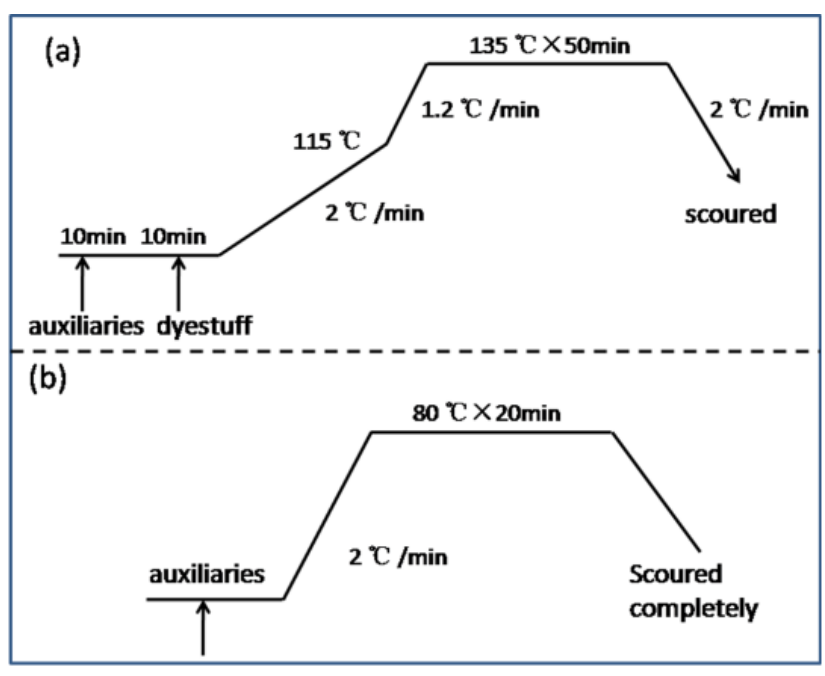

Figure 1: Dyeing (a) and scouring (b) processing profiles.

The PET woven fabrics were dyed with one formulation (Shanghai ANOKY Textile Chemicals Co., Ltd., China) that appeared to be black in visible light. The disperse dyestuff mixture (Shanghai ANOKY Textile Chemicals Co., Ltd., China) was composed of Lemon Yellow UND (1.2\%), Red ERD (1.8\%) and Blue WHRD (4.2\%). The dyeing and scouring processing is shown in Figure 1. In the dyeing process, auxiliaries (Shanghai ANOKY Textile Chemicals Co., Ltd., China) were dispersion leveler agent PES-H of $0.5 \mathrm{~g} / \mathrm{L}$, putty RE of $1.0 \mathrm{~g} / \mathrm{L}$ and buffer with $\mathrm{pH}$ values of $4 \sim 5$. In the scouring procedure, caustic soda of $1 \mathrm{~g} / \mathrm{L}$ and hydrosulfite of $3 \mathrm{~g} / \mathrm{L}$ were used. The liquor ratio (LR) in both dyeing and scouring processes was $1: 20$. The rinse pre-treatment of the fabrics before dyeing was carried out with detergent RA of $3 \mathrm{~g} / \mathrm{L}$ and $\mathrm{NaOH}$ of $2 \mathrm{~g} / \mathrm{L}$ at $95^{\circ} \mathrm{C}$ for 30 minutes. The dyeing and washing procedures of the knitted sample $\mathrm{K} 2$ were the same to that applied to the woven fabrics, except its color of blue dyed using the dyestuff purchased from the same company as mentioned above.

\section{Morphologies of fiber and fabrics}

Surface morphologies examinations of the treated NCCS PET fibers and their fabrics were performed by using a Scanning Electron Microscope (SEM). The cross-sections of the NCCS PET fibers in the yarns were obtained by using frozen section techniques [25] with a Leica CM 1950 Cryostat. Five $2 \times 2 \mathrm{~cm}^{2}$ pieces of fabric were cut in the weft direction only for all samples. The samples were cut from each fabric were then stacked in 5 layers and placed in plastic Croyomold $^{\circledR}$ (Sakura Finetek USA Inc.). A small amount of Cryo-Gel ${ }^{\mathrm{TM}}$ (Instrumedics Inc., St. Louis, MO USA) was dripped to embed and be soaked up in the 5 layer sample. The samples were then vertically placed on a specimen disc and frozen at $-20^{\circ} \mathrm{C}$ for $10 \mathrm{~min}$ to form a frozen mold. The frozen molds were transferred to a Lecia CM1950 cryostat microtome (Leica Microsystems, Bannockburn, Illinois, USA). The first sectioned piece was eliminated, but afterward ten pieces of $15 \mu \mathrm{m}$ thickness sections of each fabric were collected on plain glass slides and covered with Cytoseal ${ }^{\mathrm{TM}}$ XYL (Richard-Allan Scientific, UK). Then, the prepared slides were examined optical microscopy (ProgRes ${ }^{\circledR} 2.0$ Digital Microscopy camera, Olympus). Five trials of each were taken and recorded. 
Citation: Liu X, Wang F, Hardin IR (2013) Evaluation of Light and Color Performances of Deep Black Coloring of Non Circular Cross Section Polyester Fabrics using Polarization Image Processing. J Textile Sci Eng 4: 145. doi:10.4172/2165-8064.1000145

\section{Goniophotometer (GP) testing of single NCCS fibers and their yarns}

A Goniophotometer (GP) was used to test the reflectance of the yarn and its monofilaments arranged in horizontal and vertical mounted directions. The testing was conducted using a $632 \mathrm{~nm} \mathrm{He}-\mathrm{Ne}$ laser beam with a fixed incident angle of $45^{\circ}$ with the measurements done at $0^{\circ}$ to $90^{\circ}$ of received angle. Three levels of force were loaded on the fiber and yarn samples during testing in order to see what happens and simulate the status of the fibers/yarns in its fabrics while testing its light and color performances. Its measurement set-up is shown in Figure 2.

\section{Polarization imaging of NCCS fabrics}

A commercial system, Bossa Nova Tech [26], was used. Its optical set-up and the sample positioning are shown in Figure 3 [20]. Three types of images were recorded: a normal intensity representing what a human eye would see, a diffused light image representing the unpolarized light, which shows only the light scattered inside the fiber-the background color of the fiber, and a specular image light representing the polarized light, which shows only the reflections (first and second) that are responsible for the visual sensation of fiber luster. Angular profiles of the specular and diffused light were computed from the acquired images, as well as that of the gloss and chroma bands extracted from the specular profile. These were based on the fact that the gloss band was white while the chroma one was colored. The Lightness (L) component derived from the chroma profile was used for

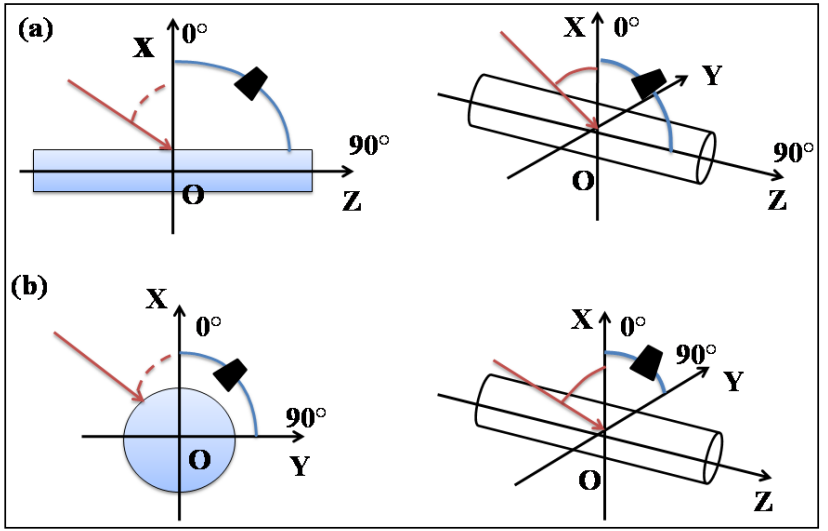

Figure 2: Schematics of horizontally (a) and vertically (b) mounted fibers/ yarns.

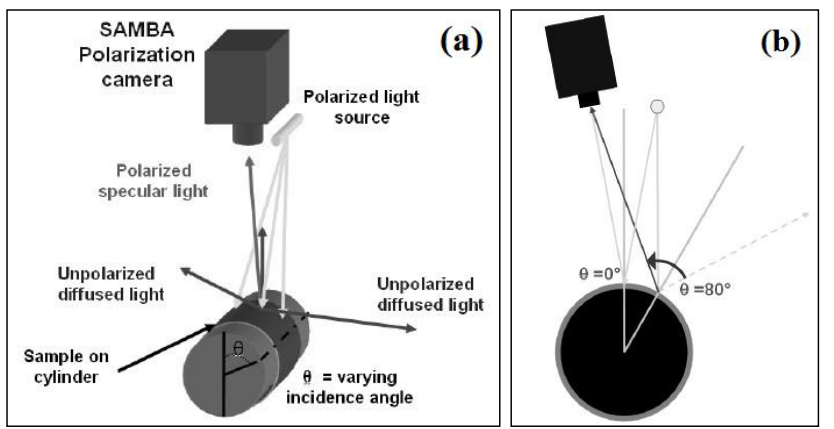

Figure 3: Schematics of horizontally (a) and vertically (b) mounted fibers/ yarns.

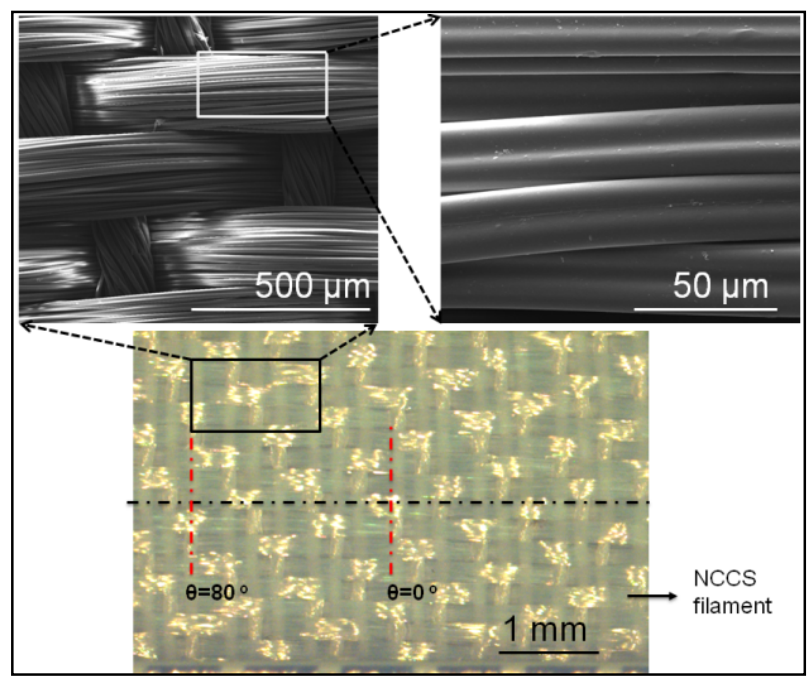

Figure 4: NCCS woven fabrics' arrangement in the polarization imaging system.

characterizing the deep-black of the fabrics dyed in black color. During the polarization imaging testing, our woven fabrics were set-up as shown in Figure 4 with the NCCS PET filament yarns in the fabrics at angle $\theta$ from 0 to $80^{\circ}$, as shown in Figure $3 \mathrm{~b}$. This is the reason that the GP testing was performed on a single NCCS fiber and its yarn on both horizontally and vertically directions.

\section{Subjective visual evaluation on deep-coloring of woven fabrics}

The testing fabrics were placed on flat white paper on a table inside a room with ambient temperature at $25 \pm 2 \%$ and $60 \pm 3 \%$ relative humidity on sunny days. The evaluators estimated the color depth of the samples and their corresponding ones under north daylight. Since there was no standard subjective assessment, 20 persons were invited at random in this experiment. Each evaluator arranged from lesser to higher color depth of the dyed samples. The lowest one was a score of 1 , the next one was 2 and the highest one was n. Finally, the total subjective score $(\mathrm{Fi})$ of the each sample was calculated by adding the scores of each evaluator, with the rank location of the sample with the lowest score marked as 1 , that of the next one as $2 \ldots$ and than that of the highest as $\mathrm{n}$. For each sample being tested, the total subjective score (Fi) could be described as Equation (1),

$$
F_{i}=\sum_{j=1}^{m=20} S S_{j i}
$$

where, $i$-the number of the samples being tested, $1,2, \ldots, \mathrm{n}$;

$j$-the number of the subjective evaluators, $1,2, \ldots, \mathrm{m}$;

$S S_{j i}$-the subjective scores of the $i$-th sample evaluated by the $j$-th evaluator.

The maximum and minimum in the rank location $\left(P_{m}\right)$ of the sample could be described as Equation (2), and the others could be shown as the similar way.

$$
P_{m}=\left\{\begin{array}{c}
\operatorname{Max}\left[F_{1}, F_{2}, \ldots, F_{n-1}, F_{n}\right]=n \\
\operatorname{Min}\left[F_{1}, F_{2}, \ldots, F_{n-1}, F_{n}\right]=1 \quad, i \in[1,2, \ldots, n](2) \\
\operatorname{Min}<\left[F_{1}, F_{2}, \ldots, F_{n-1}, F_{n}\right]<\operatorname{Max}=i
\end{array}\right\}
$$


Citation: Liu X, Wang F, Hardin IR (2013) Evaluation of Light and Color Performances of Deep Black Coloring of Non Circular Cross Section Polyester Fabrics using Polarization Image Processing. J Textile Sci Eng 4: 145. doi:10.4172/2165-8064.1000145

\section{Results and Discussion}

\section{Surface morphologies of NCCS fabrics}

The SEM images, shown in Figure 5, exemplify the surface conditions differences among the different fabric samples. These surface characteristics could affect the surface reflection, including the specular and diffused components, and then directly affect the color perceived by human eyes. However, the surface differences seen in the SEM images cannot be perceived by human eyes directly. The SEM images also confirmed the non-circular cross sections as shown in Figure 4, and strengthen the point that the contour shapes changes in fibers cross sections changes the light and color perceived in fabric and cloth. Indeed, the deep-color effect cannot be determined directly from the information provided by SEM images, but these images tell us why and how it works.

\section{Goniophotometer (GP) testing}

The goniophotometer (GP) curves of horizontally and vertically (b) mounted single fibers and those of their filaments at various tensions are shown in Figures 6 and 7, respectively. It can be seen in Figure 6 that the shape of reflection curve is profoundly influenced by the tension applied on the $\mathrm{H}$ and $\mathrm{V}$ types during the testing. However, there are not so much significant results in the yarns with the fibers. As the strain increases in a single fiber, the reflection profile tends
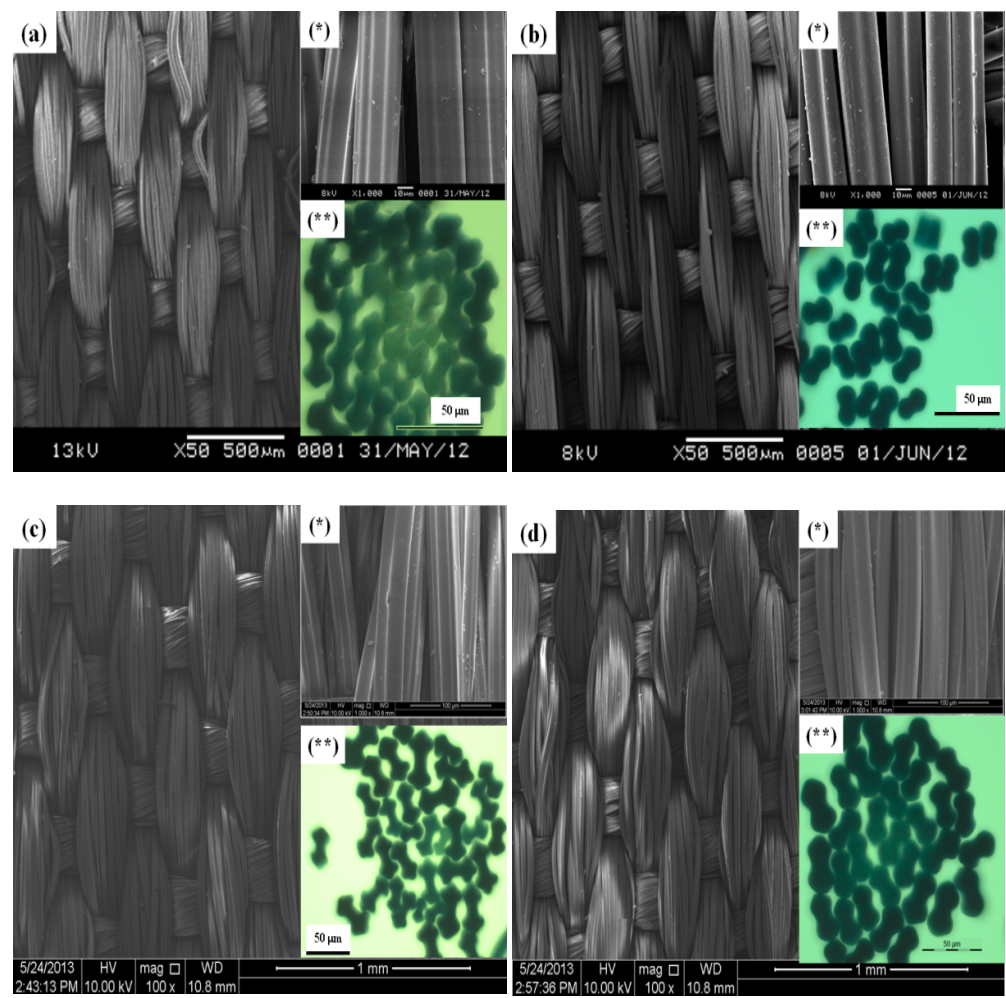

Figure 5: SEM images of the NCCS PET fabrics(a:F1, b:F2, c:F3, d:F4) and their corresponding longitudinal (*) and optical images of the fiber's cross sections $\left.{ }^{* *}\right)$.
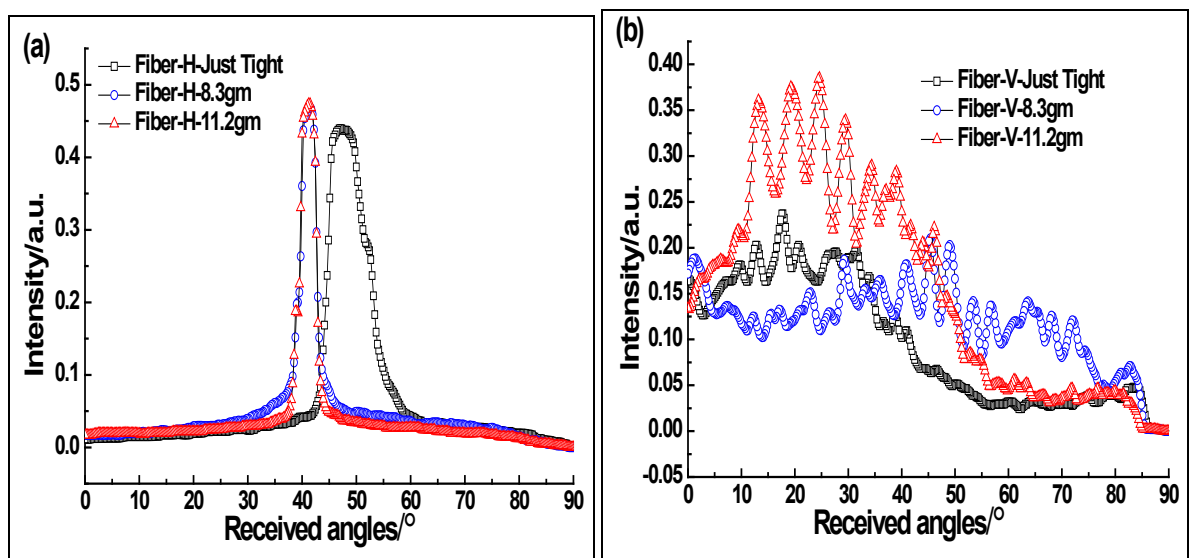

Figure 6: Horizontally (a) and vertically (b) mounted single fibers with varied tension. 

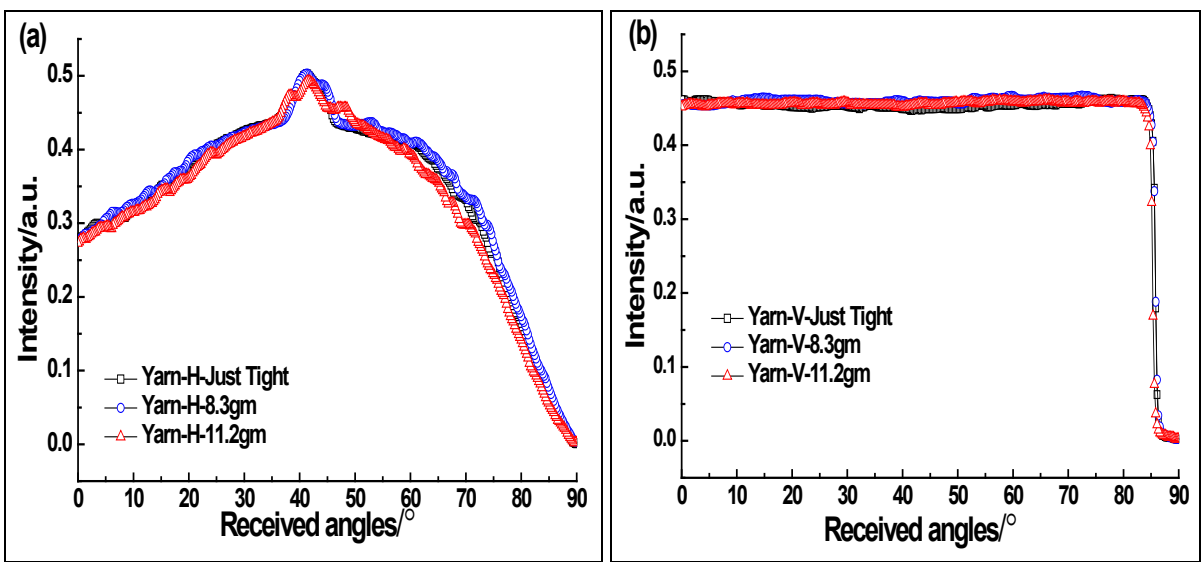

Figure 7: Horizontally (a) and vertically (b) mounted yarn with varied tension.
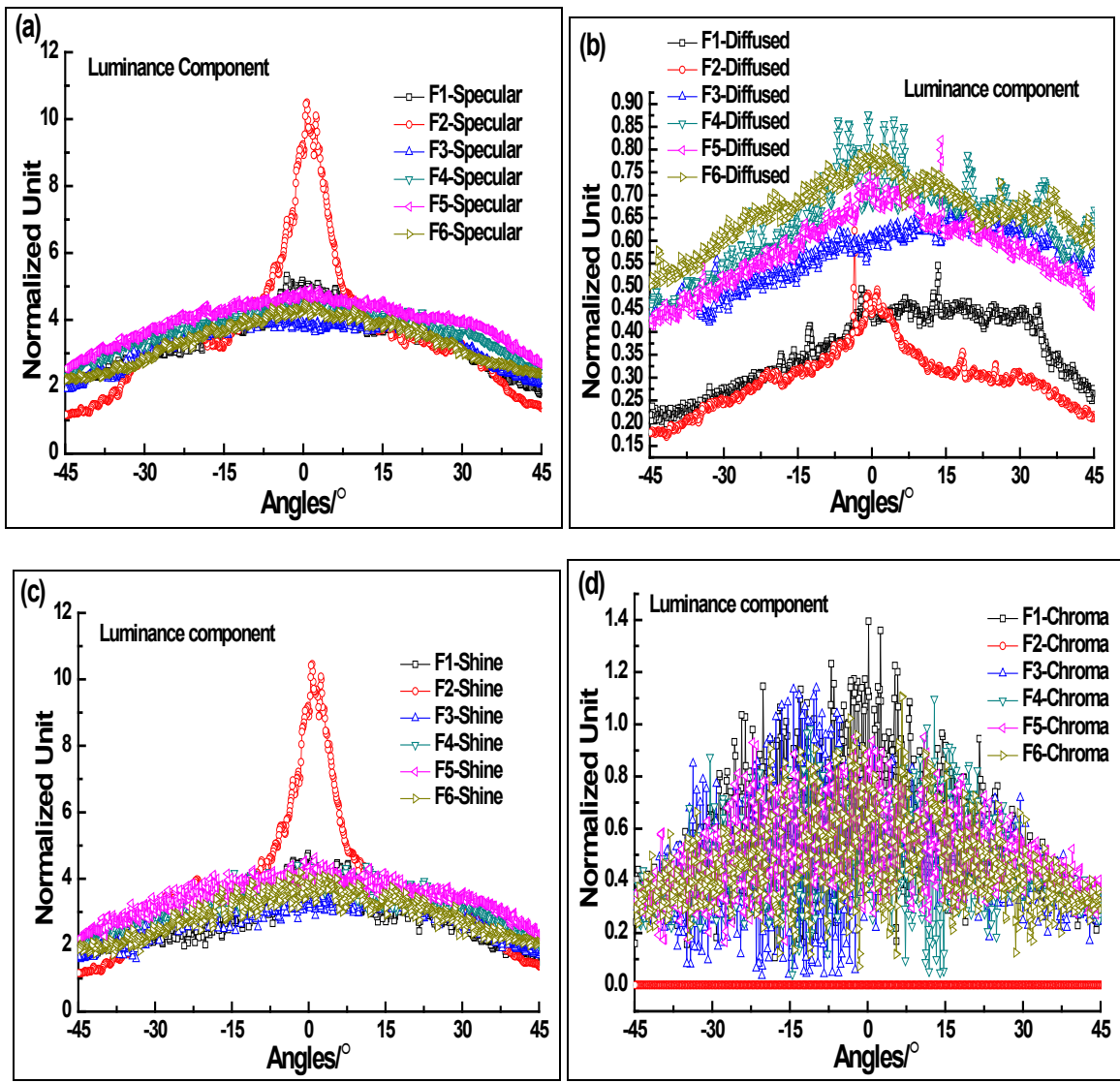

Figure 8: Angular distribution of the images on black-dyed woven NCCS PET fabrics: (a) specular, (b) diffused,(c) shine,(d) chroma.

more towards specular reflection due to the surface of the fiber being flattened out from the force loaded. However, the reflection profiles for both horizontally and vertically mounted yarn show no difference among the samples as loading is increased. While a peak occurred in the horizontally-type GP testing, there was not one for the vertically mounted one. This is difficult to explain.

\section{Polarization imaging processing}

The polarization imaging processed results of the interwoven and knitted fabric samples are shown in Figure 8 and Figure 9, respectively. The specular, diffused, and shine as well as its chroma component of its luminance aspect were described in these figures.

As can be seen from Figure 8a and 8c, the specular and shine distributions of the F2 was much different from the others, this was confirmed by the images recorded in Figure 10. But there were no significant differences among the other five specimens in these two aspects. Neither were there significant differences in the chroma of all 

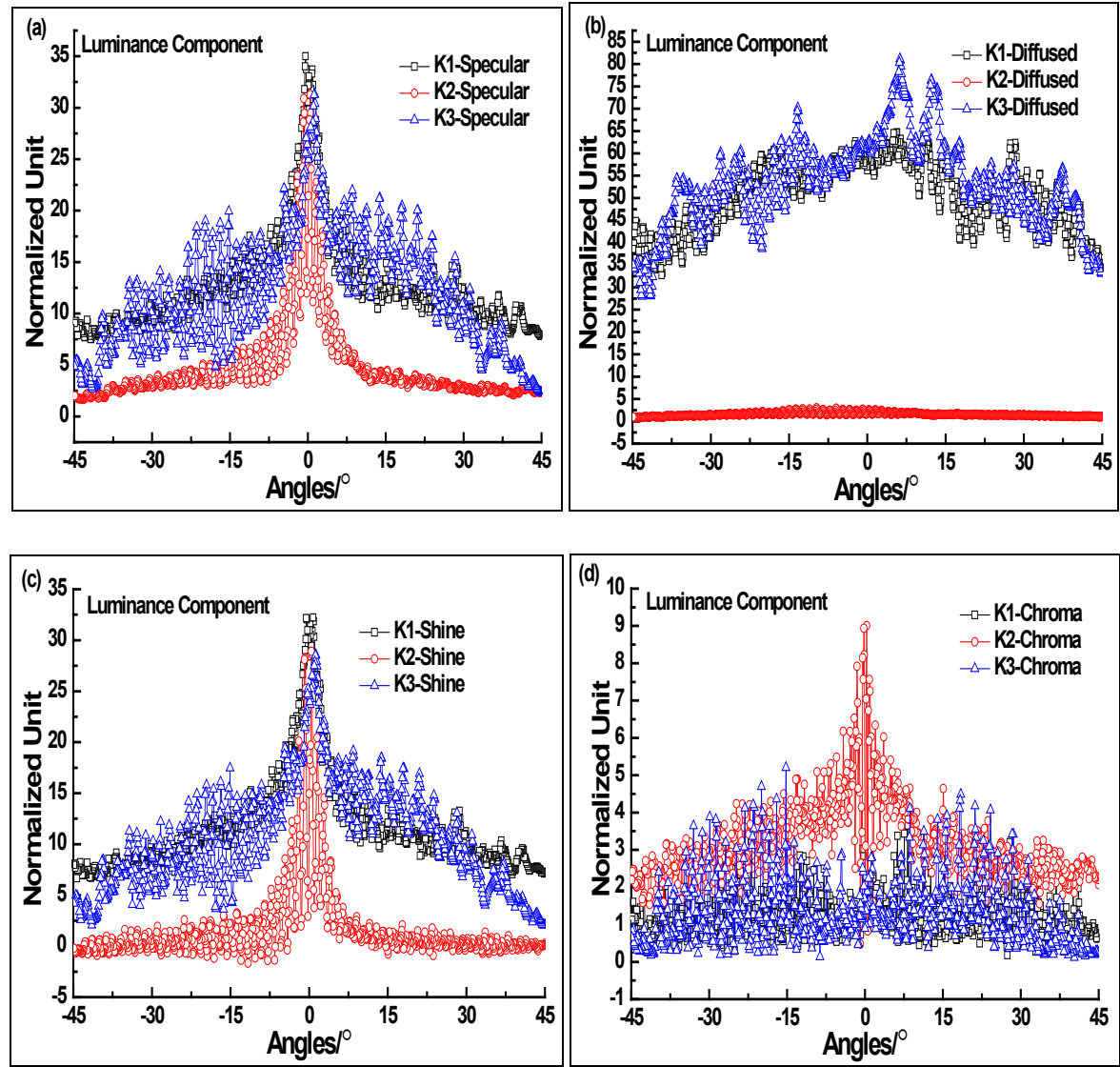

Figure 9: Angular distribution of the images of the dyed and undyed knitted fabrics: (a) specular, (b) diffused, (c) shine, (d) chroma.

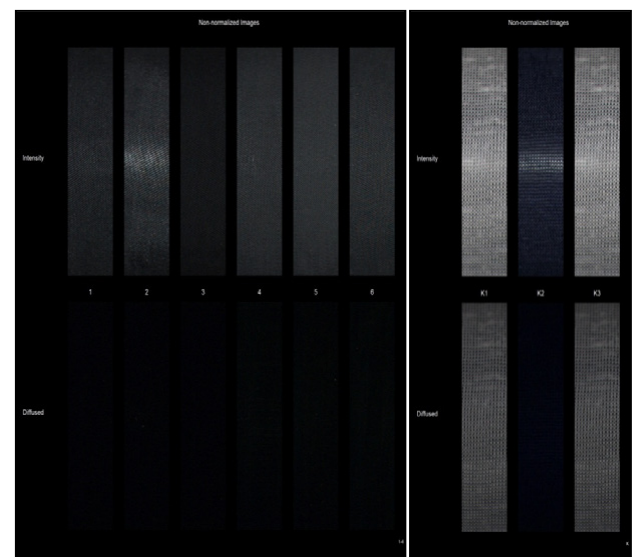

Figure 10: The processed non-normalized images of the interwoven (a) and knitted (b) fabrics: Intensity (top) and Diffused (bottom).

the samples. Meanwhile, Figure $8 \mathrm{~b}$ shows that different woven fabric samples had different diffused light that could be perceived even though they were dyed the same way. This may be caused by the fiber with different cross-section shapes. Figure $8 \mathrm{~d}$ indicated that the color of these 6 woven fabrics was true because the chroma values were so overlapped that they could not be distinguished from each other according their angular distribution curves. The exception to this was F2 with no chroma value due to its much higher shine and specular values.
The curve shapes in Figure 9a for specular values was similar to that of Figure $9 \mathrm{c}$ in shine values, which means that the specular component contributed much to the shine values of the fabrics. There are peaks occurring around $0^{\circ}$ for both the specular and shine angular distributions. There is not a similar trend in either the diffused or chroma angular distributions. In Figure 9c the two white samples K1 and $\mathrm{K} 3$ show much higher diffused light component, but there is none in K2. This may be due to its dark color compared to the white knitted samples. However, higher chroma values in K2 compare to those in 

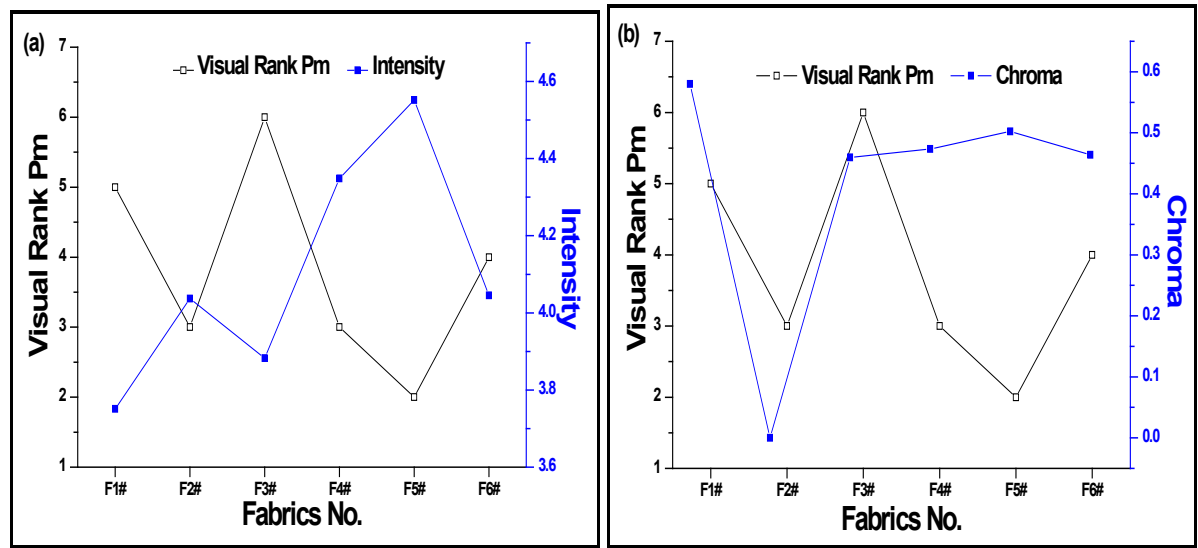

Figure 11: Relationships of the visual rank Pm to the Intensity (a) and Chroma (b) values.

\begin{tabular}{|c|c|c|c|}
\hline Fabrics No. & $\begin{array}{c}\text { Visual rank Pm of } \\
\text { Intensity* }\end{array}$ & \multicolumn{2}{|c|}{$\begin{array}{c}\text { Mean values } \\
\text { calculated** }\end{array}$} \\
\cline { 3 - 4 } & & Intensity & Chroma \\
\hline F1" & 5 & 3.7509 & 0.58 \\
\hline F2 & 3 & 4.037 & 0 \\
\hline F3" & 6 & 3.8825 & 0.4598 \\
\hline F4" & 3 & 4.3487 & 0.4736 \\
\hline F5 & 2 & 4.5516 & 0.5021 \\
\hline F6" & 4 & 4.0451 & 0.464 \\
\hline
\end{tabular}

*Visual evaluation was conducted on the images corresponding to the Intensity and diffused one of Figure 10.

**Inthepolarizationimaging, Specular=Shine+Chroma, Intensity=Specular+Diffused [20], here the L component of each were used in this analysis.

Table 2: Visual evaluated rank location $P_{m}$ and the mean values calculate.

white samples $\mathrm{K} 1$ and $\mathrm{K} 3$. Both Figure $9 \mathrm{~b}$ and $9 \mathrm{~d}$ indicate that there are not so many differences in diffused and chroma values as might be expected from the fineness differences in the fibers as well as in their filament yarns. The color dyed will affect the diffused and chroma values much more significantly than the specular and shine values. One interesting thing that can be seen from Figure $9 \mathrm{~b}$ is that the diffused value of the K2 sample was close to zero at all angles. This might be due to its dark color compared to the two white knitted samples. Meanwhile, the chroma value of the $\mathrm{K} 2$ sample at all angles was a little more than those of the other two samples.

The non-normalized images of the polarization procedure for the woven and knitted fabrics are shown in Figure 10. All of these images recorded the normal intensity and its corresponding diffused reflection, the former of which represents what human eyes could see and the latter one showing the depolarized light. The subjective perceived colors seem different from the results indicated from Figure 8 to Figure 10. This may be caused by the mechanism of perception in the objective apparatus and the human organism. In addition, there are personal experience and emotional factors involved in human eye subjective evaluation procedure. But the trend suggested by the objective tested indexes was true for its comparison.

\section{Visual evaluation}

All six samples were presented to judges in random order. The rank locations $\left(P_{m}\right)$ of all the six samples are shown in Table 2, as well as their calculated corresponding mean values of intensity and chroma in polarization imaging testing results. Figure 11 showed the relationships of visual rank location $\left(P_{m}\right)$ to the mean Intensity and
Chroma values. Subjective visual evaluation of light and color of woven fabrics is a comprehensive procedure in the human brain. Light falls on the colorants (dyes and pigments) and this causes the white light to be broken into its component wavelengths. Reflected light also results from the real three-dimensional nature of fabrics and yarns. There is a multi-sensory, emotional and psychological experience involved which is based on participants' reality of preference, personality and emotion in this process. This is also happens when a consumers making a decision in buying cloth or a garment. The amount and direction of reflectance that can be perceived by human eyes is in turn responsible for the perceived value of the fabric color. That means that the high level of total light reflectance results in a high value (light color) while a low level indicates a low value (or dark color). If light from a surface is organized and reflected in a single direction, the surface appears either very light if it is reflecting toward the viewer or dark if its reflecting away from the viewer.

As can be seen from Figure 11a, it cannot be concluded from the comparison between the overall visual evaluation of the intensity images and the average values of intensity. One reason might be that human subjective evaluation will mainly affect the specular and shine appeared on the surface of the fabrics, which can be confirmed by sample of F2\# and F6\#. Another reason may be the mechanism of human eyes and the machine we used for perceiving the surface reflectance of the samples illuminating by polarized light directly. Figure $11 \mathrm{~b}$ showed clearly that there is a higher consistency of the visual rank Pm to the values of chroma on discussing the deep-coloring of NCCS PET fabrics when compare to that in fabrics with round PET fibers. This may be contributing to the lower values of specular and shine values of fabrics made of NCCS fibers. This also indicated that color will be changed by using some PET fibers with special cross-sections, and shown same trend between subjective evaluation and objective testing results.

\section{Conclusion}

The light and color performance on deep-black-coloring of fabrics made of NCCS PET were investigated by using GP and polarization imaging. The GP testing results showed that the surface reflectance of single NCCS fiber were profoundly affected by the forces loaded under horizontally and vertically directions compare to its yarns. The fibers' cross-section and fabrics' SEM images showed that the unique surface characters of the NCCS PET will give unpredictable effects on the light and color perceived of the fabrics. The polarization imaging processed results of black-dyed woven fabrics indicated that higher 
Citation: Liu X, Wang F, Hardin IR (2013) Evaluation of Light and Color Performances of Deep Black Coloring of Non Circular Cross Section Polyester Fabrics using Polarization Image Processing. J Textile Sci Eng 4: 145. doi:10.4172/2165-8064.1000145

specular and chroma values will affect its deep-coloring effect, and the overlapped chroma curves means insignificant difference among those black fabrics. Meanwhile the polarization imaging results of the knitted fabrics suggested that higher value of chroma means higher effect of deep-coloring compare to undyed/white samples with higher values of specular and shine. Subjective visual evaluation was carried on the postprocessed polarization images of NCCS PET woven samples and made comparison with that of the instrumental ones, and the results revealed that there is a good consistency on deep-coloring perceived between the intensity images and average value of chroma while inconsistency occurred between the intensity images and average value of intensity.

\section{Acknowledgement}

The research was financially supported by the Fundamental Research Funds for the Central Universities [grant No. 12D10137] as well as partial financial support from the China Scholarship Council (CSC). The authors thank Dr. Ram Ramaprasad and Ms. Catherine Qi from the Textile Research Institute (TRI, Princeton, NJ, USA) for their help in Goniophotometer testing. The authors also would like to express their thanks to Mr. Nick Lechocinski (Bossa Nova Technologies, Culver City, CA

USA) for his efforts on collecting the data on polarization imaging used in this paper. The authors also like to thank Ms. Rachel C. Steffens (Virology Technician, Athens Veterinary Diagnostic Laboratory at the University of Georgia for her help on cutting fiber cross-sections.

\section{References}

1. Roy SB, Li XM (2002) Principle of Color (1st edn.). Materials Science and Engineering Center Press, Beijing, China.

2. Jameson KA, Highnote SM, Wasserman LM (2001) Richer color experience in observers with multiple photopigment opsin genes. Psychon Bull Rev 8: 244261.

3. Deeb SS (2005) The molecular basis of variation in human color vision. Clin Genet 67: 369-377.

4. Pardo PJ, Perez AL, Suero MI (2007) An example of sex-linked color vision difference. Color Research and Application 32:433-439.

5. Hutchings JB (2002) The perception and sensory assessment of colour, In MacDougall DB. Colour in Foods: Improving Quality. Woodhead, Cambridge, England.

6. Allen EH, Goldfinger G (1972) The color of absorption scattering substrates: I. The color of fabrics. J Appl Polymer Sci 16: 2973-2982.

7. Goldfinger G, Lau KC, McGregor R (1973) The effect of the distribution of colorant on the color of fibers. J Appl Polymer Sci 11: 481-484.

8. Goldfinger G, Lau KC, McGregor R (1974) The effect of the distribution of colorant on the color of fibers. J Appl Polymer Sci 18:1741-1751.
9. Allen EH, Goldfinger G (1973) The effect of ring dyeing on the color of fibers. J Appl Polymer Sci. 17: 1627-1629.

10. Goldfinger G, Wiggs JH (1978) The effect of internal scattering on the color of fabrics. J Appl Polymer Sci. 22: 3459-3468.

11. Dominick AB (1990) Effect of fiber translucency on the color of blends of precolored fibers. Textile Res J 60: 162-167.

12. Kobsa H, Rubin B, Shearer SM (1993) Using optical ray tracing to explain the reduced dye yield of microdenier yarns. Textile Res J 63: 475-479.

13. Tsoutseos AA, Nobbs JH (2000) Alternative approach to color appearance of textile materials with application to the wet/dry reflectance prediction. Textile Chemist and Colorist Am Dyestuff Reporter 32: 38-43.

14. Dorsch P, Wilsing H, Peters KH (1981) Parameters influencing the depth of dyed shades, particular on Acrylic fibres. Melliand Textilberichte. 62: 188-192.

15. Steinlin F (1982) Connection between fibre cross-section and the shade dyed on man-made fibres. Melliand Textilberichte. 64:213-216.

16. Bustard HK, Smith RW (1991) Investigation into the scattering of light by human hair. Appl Opt 30: 3485-3491

17. Stamm RF Garcia MK, Fuchs JJ (1977) The optical properties of human hair I. fundamental considerations and goniophotometer curves. J Soc Cosmet Chem 28: $571-600$.

18. Stamm RF Garcia MK, Fuchs JJ (1977) The optical properties of human hair II. The luster of hair fibers. J Soc Cosmet Chem 28:601-609.

19. Guiolet A, Garson JC, Levecque JL (1987) Study of the optical properties of human hair. Int J Cosmet Sci 9: 111-124.

20. Lefaudeux N, Lechocinski N, Clemenceau P, Breugnot S (2009) New luster formula for the characterization of hair tresses using polarization imaging. $J$ Cosmet Sci 60: 153-169.

21. Gao T, Landa P, Tillou R (2012) Hair color vibrance factor to characterizes shine and color intensity. Cosmetics and Toiletries magazine. 127: 48-56.

22. Lechocinski N, Breugnot S (2011) Fiber orientation measurement using polarization imaging. J Cosmet Sci 62: 85-100.

23. Breugnot S, Lechocinski N, Pouet BF (2012) Visual appearance measurement method and system for randomly and regularly arranged birefringent fibers. US Patent No. 8223322 B2, USA

24. Yu XF (2004) Experimental Technology of Textile Materials. Beijing: China Textile Press, China.

25. Befru TR, Ayse O, Remziye D te al. (2011) Predicting the intra-yarn porosity by image analysis method. Textiles Res J 82: 1720-1728.

26. http://www.bossanovatech.com/samba_hair_system.htm 